\title{
Dietary fibre (DF) and NSP intake in Irish teenagers aged 13-17 years
}

\author{
S. Bannon, J. Walton, E. M. Hannon and A. Flynn \\ Department of Food and Nutritional Sciences, University College Cork, Cork, Republic of Ireland
}

The objectives of the present study were to measure DF and NSP intakes in Irish teenagers aged 13-17 years and the contribution of food groups to DF intake. Data from the National Teens' Food Survey (NTFS) was used for this purpose. The NTFS was carried out between September 2005 and 2006 to establish a database of habitual food and drink consumption of a representative sample of Irish teenagers aged 13-17 years. A $7 \mathrm{~d}$ semi-weighed food record was used to collect food data from 441 teenagers ( 224 males; 217 females). Analysis of dietary intake data was carried out using WISPC (Tinuviel Software, Llanfechell, Anglesey, UK), which is based on McCance and Widdowson's The Composition of Foods, Sixth Edition ${ }^{(1)}$.

Mean daily DF and NSP (g and g/10 MJ energy) intakes are reported. For both age-groups mean daily DF and NSP intakes were significantly $(P<0.001)$ higher for males compared with females. Intakes increased across age-groups for both DF and NSP. However, there were no significant differences in fibre density as DF or NSP across age-groups or between genders.

\begin{tabular}{|c|c|c|c|c|c|c|c|c|}
\hline & \multicolumn{4}{|c|}{ Males } & \multicolumn{4}{|c|}{ Females } \\
\hline & \multicolumn{2}{|c|}{$13-14$ years $(n 95)$} & \multicolumn{2}{|c|}{$15-17$ years $(n 129)$} & \multicolumn{2}{|c|}{$13-14$ years $(n 93)$} & \multicolumn{2}{|c|}{$15-17$ years $(n 124)$} \\
\hline & Mean & SD & Mean & SD & Mean & SD & Mean & SD \\
\hline DF: $g / d$ & $16.5^{*}$ & 5.5 & $18.5^{*}$ & 9.0 & 12.9 & 4.0 & 13.6 & 4.5 \\
\hline $\mathrm{g} / 10 \mathrm{MJ}$ per $\mathrm{d}$ & 18.4 & 5.0 & 18.6 & 6.1 & 18.4 & 3.6 & 19.6 & 6.0 \\
\hline NSP: g/d & $12.3^{*}$ & 4.1 & $13.7 *$ & 7.1 & 9.7 & 3.1 & 10.3 & 3.5 \\
\hline $\mathrm{g} / 10 \mathrm{MJ}$ per $\mathrm{d}$ & 13.8 & 4.0 & 13.8 & 5.0 & 13.7 & 2.8 & 14.8 & 4.7 \\
\hline
\end{tabular}

Values were significantly higher than those for females in same age-group: $* P<0.001$.

The contribution of food groups ( $\mathrm{g}$ and \%) to mean daily DF intakes by age and gender are reported.

\begin{tabular}{|c|c|c|c|c|c|c|c|c|}
\hline & \multicolumn{4}{|c|}{ Males } & \multicolumn{4}{|c|}{ Females } \\
\hline & \multicolumn{2}{|c|}{$\begin{array}{c}\text { 13-14 years } \\
(n \text { 95) }\end{array}$} & \multicolumn{2}{|c|}{$\begin{array}{c}\text { 15-17 years } \\
(n \text { 129) }\end{array}$} & \multicolumn{2}{|c|}{$\begin{array}{c}\text { 13-14 years } \\
(n 93) \\
\end{array}$} & \multicolumn{2}{|c|}{$\begin{array}{c}15-17 \text { years } \\
(n 124)\end{array}$} \\
\hline & $\%$ & $\mathrm{~g}$ & $\%$ & $\mathrm{~g}$ & $\%$ & $\mathrm{~g}$ & $\%$ & $\mathrm{~g}$ \\
\hline Bread and rolls & 22.5 & 3.6 & 22.8 & 4.3 & 19.7 & 2.5 & 22.5 & 3.1 \\
\hline Potatoes and potato products & 19.5 & 3.1 & 18.3 & 3.0 & 20.4 & 2.4 & 19.4 & 2.5 \\
\hline Vegetablesh and vegetable dishes & 11.2 & 1.9 & 10.9 & 2.1 & 11.5 & 1.6 & 11.6 & 1.6 \\
\hline Breakfast cereals & 13.4 & 2.3 & 13.6 & 2.7 & 8.0 & 1.1 & 8.4 & 1.3 \\
\hline Grains, rice, pasta and savouries & 10.4 & 1.7 & 9.9 & 1.6 & 10.3 & 1.3 & 9.3 & 1.2 \\
\hline Sugars, confectionery, preserves and savoury snacks & 6.4 & 1.1 & 5.6 & 1.0 & 10.6 & 1.3 & 9.0 & 1.1 \\
\hline Fruit and fruit juices & 5.3 & 1.0 & 6.3 & 1.6 & 7.2 & 1.0 & 8.8 & 1.3 \\
\hline Meat and meat products & 4.9 & 0.8 & 5.5 & 0.9 & 5.5 & 0.7 & 4.2 & 0.5 \\
\hline Other food groups & 6.5 & 1.0 & 7.0 & 1.3 & 6.9 & 0.9 & 6.8 & 0.9 \\
\hline Total & 100 & 16.5 & 100 & 18.5 & 100 & 12.8 & 100 & 13.6 \\
\hline
\end{tabular}

A significantly $(P<0.001)$ higher percentage of females $(13-14$ years, 91; 15-17 years, 93) compared with males $(13-14$ years, 68; 15-17 years, 72) did not meet the American Health Foundation recommendation ${ }^{(2)}$ of a DF intake (g) of $\geq$ age +5 .

The project was funded by the Irish Government under the National Development Plan 2000-2006.

1. Food Standards Agency (2002) McCance \& Widdowson's The Composition of Foods Sixth Edition. Cambridge: Royal Society of Chemistry.

2. Williams CL, Bollella M \& Wynder EL (1995) Pediatrics 96, 985-988. 